\title{
Super-resolution microscopy: successful applications in centrosome study and beyond
}

\author{
Jingyan $\mathrm{Fu}^{1 凶}$, Chuanmao Zhang ${ }^{2 \bowtie}$ \\ 1 State Key Laboratory of Agrobiotechnology, College of Biological Sciences, China Agricultural University, \\ Beijing 100193, China \\ 2 Key Laboratory of Cell Proliferation and Differentiation of the Ministry of Education, College of Life Sciences, \\ Peking University, Beijing 100871, China
}

Received: 16 August 2019 / Accepted: 30 September 2019 / Published online: 21 November 2019

\begin{abstract}
Centrosome is the main microtubule-organizing center in most animal cells. Its core structure, centriole, also assembles cilia and flagella that have important sensing and motility functions. Centrosome has long been recognized as a highly conserved organelle in eukaryotic species. Through electron microscopy, its ultrastructure was revealed to contain a beautiful nine-symmetrical core 60 years ago, yet its molecular basis has only been unraveled in the past two decades. The emergence of super-resolution microscopy allows us to explore the insides of a centrosome, which is smaller than the diffraction limit of light. Super-resolution microscopy also enables the compartmentation of centrosome proteins into different zones and the identification of their molecular interactions and functions. This paper compiles the centrosome architecture knowledge that has been revealed in recent years and highlights the power of several super-resolution techniques.
\end{abstract}

Keywords Centrosome, Super-resolution microscopy, SIM, STED, SMLM

\section{INTRODUCTION}

Centrosome was discovered in the late nineteenth century during the first study of mitosis. Centrosome forms the poles of the mitotic spindle towards which chromosomes migrate (Fig. 1). Although the presence of supernumerary centrosomes in cancer cells was discovered around the same time, the importance of these centrosomes remains poorly understood (Godinho and Pellman 2014; Nigg and Holland 2018). At its core, a centrosome has a nine-fold symmetrical centriole, which also acts as the basal body of the cilium. Cilia are hair-like protrusions found at the surface of most eukaryotic cells. Motile cilia can beat in a coordinated fashion to generate fluid flow or locomotion, whereas non-motile cilia transmit signals from the extracellular

$\bowtie$ Correspondence: jingyanfu@cau.edu.cn (J. Fu), zhangcm@pku.edu.cn (C. Zhang) milieu to the cell nucleus. Mutations in centrosome proteins can be found in a wide range of human genetic diseases, including microcephaly, and a group of disorders that are collectively known as ciliopathies, such as retinal degeneration, renal failure, cystic kidneys, obesity, liver dysfunction, and neurological disorders (Chavali et al. 2014; Fu et al. 2015; Nigg and Holland 2018).

Understanding how centrosome is assembled and regulated is important in unraveling the mechanistic basis of relevant human diseases. In line with DNA replication, a centrosome duplicates once every cell cycle (Firat-Karalar and Stearns 2014; Fu et al. 2015; Nigg and Holland 2018). A newly born cell has two centrioles, of which one is engaged orthogonally to the other. This arrangement is lost as these centrioles disengage in early G1 and then wander apart while being linked by a loose fibrous connection. The assembly of a pro-centriole (daughter centriole) perpendicular to each 

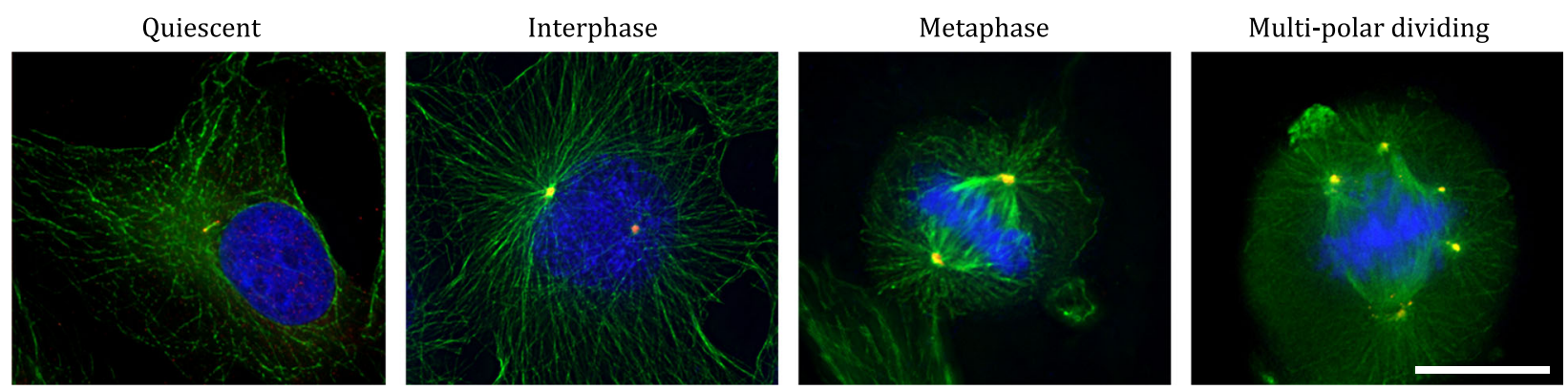

Green: microtubules / Blue: DNA / Red: cilium or centrosome

Fig. 1 Centrosome in various cell activities. From left to right: quiescent cell with a cilium, interphase, metaphase, and multi-polar dividing cell. Green, microtubules revealed by the $\alpha$-tubulin antibody; Blue, DNA; Red, cilium revealed by the IFT88 antibody (left panel) or centrosome revealed by the $\gamma$-tubulin antibody (three right panels). Scale bar, $10 \mu \mathrm{m}$

mother then begins in $\mathrm{G} 1 / \mathrm{S}$, and the pro-centrioles subsequently elongate throughout G2 until their size becomes similar to that of their mothers. Before mitosis, the mother centrioles begin to accumulate more pericentriolar material (PCM) and nucleate increased microtubules in preparation for the spindle assembly. The fibrous tether between centrosomes resolves allowing these centrosomes to disjoin and separate in opposite sides of the cell as spindle poles. In vertebrate cells, the new mother centriole assembles distal and subdistal appendages that protrude from the distal periphery of the microtubule wall (Uzbekov and Alieva 2018). Subdistal appendages have varying numbers and participate in the nucleation of microtubules, whereas distal appendages are nine-bladed, pinwheel-like structures that are essential for ciliogenesis and mediate the membrane docking and subsequent fusion of ciliary vesicles with the cytoplasmic membrane. During ciliogenesis, the centriole is also called a basal body, from which the transition zone and ciliary axoneme are built (Zhang et al. 2015, 2017, 2019).

Previous proteomic analyses and genome-wide RNAi screens have identified numerous modulators for centrosome assembly (Andersen et al. 2003; Balestra et al. 2013; Dobbelaere et al. 2008; Goshima et al. 2007; Jakobsen et al. 2011; Muller et al. 2010), but the roles of these proteins and how they fit together remain unknown. One challenge in addressing these questions is that the size of a centrosome reaches the diffraction limit for conventional light microscopy. Over the past decade, various forms of super-resolution microscopy have been proposed to address this challenge and to enable the study of these tiny structures. With these advancements, researchers have begun to study how the centrosome changes its protein compositions during various cell activities and development, thereby opening an avenue for exploring how centrosomes go awry in cancer and several heritable diseases.

\section{D STRUCTURED ILLUMINATION MICROSCOPY (3D-SIM)}

Structured illumination microscopy (SIM) is a wide-field microscopy technique that increases the spatial resolution by a factor of two compared with conventional optical microscopy (Gustafsson 2000; Gustafsson et al. 2008). SIM works by using a patterned illumination to excite the object; the striped pattern is modulated multiple times in its phases and angles, and the emitted fluorescence signal from the same object is recorded. The interaction between the excitation and object produces moiré patterns due to the interference resulting from the overlying of two grids at a specified angle. Moiré patterns contain high-frequency spatial information that would otherwise not be detected by conventional imaging. This additional information can be extracted from the raw data of multiple images by using a computational algorithm to reconstruct the final image. 3D-SIM has lateral and axial resolutions of approximately 100 and $300 \mathrm{~nm}$, respectively, which represent an eightfold increase in 3D volume.

3D-SIM has several advantages over other superresolution methods (Demmerle et al. 2017; Fiolka et al. 2012; Heintzmann and Huser 2017; Shao et al. 2011; Wu and Shroff 2018). First, 3D-SIM can be immediately used for slides that are prepared for conventional microscopes. Second, 3D-SIM can detect four wavelengths in the same sample by using standard fluorescent proteins, dyes, or a combination of both. Third, 3D optical sectioning can be easily carried out in 3D-SIM, thereby enhancing the resolution in both lateral and axial directions. Fourth, given that this technique is implemented in a wide-field microscope, 3D-SIM has a relatively high speed, thereby making this technique better suited for live cell imaging compared with other super-resolution methods. However, in 3D-SIM, the 
image reconstruction can produce artifacts that require a significant amount of knowledge from users to detect.

3D-SIM has been widely applied in centrosome study for three reasons. First, for those proteins that localize to the outer zones of the centrosome (from zone II outward, Fig. 2), their staining can be resolved into shapes instead of blobs under conventional microscopy (Fig. 3A) (Baumann 2012; Fu and Glover 2012; Gopalakrishnan et al. 2011; Lawo et al. 2012; Luders 2012; Mennella et al. 2012; Sir et al. 2011; Sonnen et al. 2012). The Drosophila centrosome thus can be divided into five major zones (Fig. 3A) (Fu and Glover 2012; Fu et al. 2016). Zone I includes proteins that take a dot shape when viewed under 3D-SIM resolution, such as the cartwheel components Sas6 and Ana2 (Dzhindzhev et al. 2014; Mennella et al. 2012). Zone II proteins have a toroid shape with a diameter of no more than $200 \mathrm{~nm}$, including Sas4, Spd2, Polo, and the centriolar microtubule wall (Fu and Glover 2012; Gopalakrishnan et al. 2011; Mennella et al. 2012). Zones I and II constitute the centriole structure, whereas zones III and IV are PCM regions. The zone III proteins Asl and Dplp are recruited to the pro-centriole in the metaphase, whereas $\mathrm{Cnn}$ and $\gamma$-tubulin are loaded in the following interphase before expanding into zone IV along with Spd2 to form a mass of PCM at the beginning of mitosis. Zone $\mathrm{V}$ proteins include CP110 and its partners that decorate the distal end of the centriole. Similarly, the PCM of the human centrosome is resolved into various concentric rings (Lawo et al. 2012; Sonnen et al. 2012). In interphase, Cep152, Cep192, PCNT, CDK5RAP2, TUBG1, and NEDD1 (homologues of Drosophila Asl, Spd2, Dplp, Cnn, $\gamma$-tubulin, and DGp71WD, respectively) occupy a space similar to zone III of the Drosophila centrosome, whereas during mitosis, Cep192, PCNT, CDK5RAP2, TUBG1, and NEDD1 expand into the PCM matrix similar to the zone IV proteins of the Drosophila centrosome. Several proteins have an elongated conformation. For instance, the C-terminus of Drosophila Cep135 occupies

A

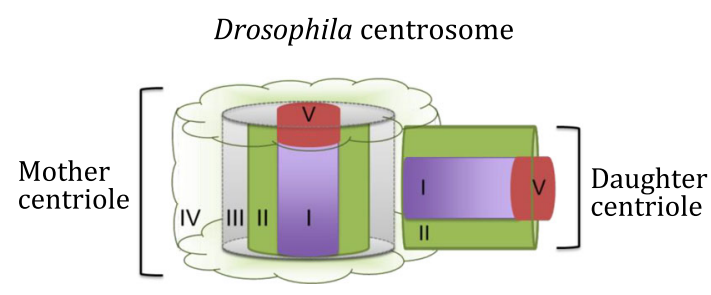

Zone I-II: Core centriole; III-IV: PCM; V: Distal end a dot in zone I, its N-terminus occupies a ring at the border of zones I and II, the N-terminus of Ana1 occupies a relatively smaller ring at the border of zones I and II, its C-terminus occupies a ring in zone II, and the $\mathrm{C}$-terminus of Asl is in zone II while its $\mathrm{N}$-terminus is in zone III (Fu et al. 2016). These three proteins form a complex through spatially overlapping regions and are necessary for newly born centrioles to duplicate and recruit PCM in both Drosophila and human cells (Chang et al. 2016; Fu et al. 2016; Izquierdo et al. 2014; Saurya et al. 2016; Tsuchiya et al. 2016). Another example is Dplp and its human homologue PCNT, whose C-terminus is distributed in a ring that overlaps with the centriolar microtubule wall in zone II and whose $\mathrm{N}$-terminus extends into the PCM in zone III in interphase (Mennella et al. 2012). Dplp is required for the initial recruitment and proper organization of mitotic PCM but is not necessary for PCM expansion; PCNT depletion affects the toroid shapes of several PCM proteins, including NEDD1, CDK5RAP2, and Cep192 (Lawo et al. 2012; Mennella et al. 2012).

Second, the mother and daughter centrioles can be well distinguished by 3D-SIM in order to trace the protein composition at each centriole separately. Previous studies have taken advantage of this capability to extensively investigate the recruitment of proteins onto newly born daughter centrioles (Dzhindzhev et al. 2014; $\mathrm{Fu}$ et al. 2016). For instance, Drosophila Ana2 is recruited to the pro-centriole earlier than Sas6, and the phosphorylation of Ana2 by Plk4 is required for its interaction with and recruitment of Sas6 (Dzhindzhev et al. 2014, 2017). Cep135 and Ana1 are sequentially recruited to the daughter centriole within a very close time window, whereas Asl and Dplp are recruited sequentially only in mitosis (Fu et al. 2016; Mennella et al. 2012). In U20S cells, the pre-replication complex component Cdc6 is recruited to the pro-centriole to prevent centriole over-duplication via the sequestering of Sas6 (Xu et al. 2017).

B

Human mother centriole

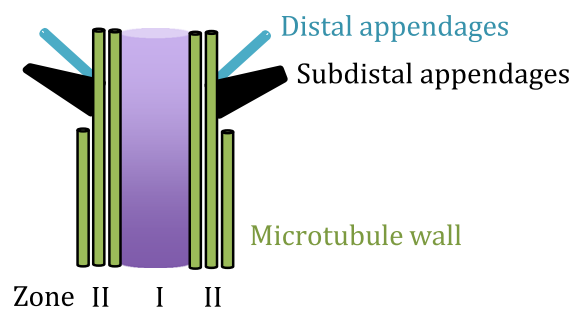

Fig. 2 Centrosome zones revealed via super-resolution microscopy. A Schematics of the Drosophila centrosome highlighting the five major zones revealed via 3D-SIM microscopy. B Human mature centriole highlighting the microtubule wall, subdistal appendages, distal appendages, and the corresponding zones I and II. The PCM compartment is not shown 
Fig. 3 Representative images obtained from 3D-SIM, STED, and SMLM. A Comparison between conventional microscopy and 3D-SIM. The 3D-SIM revealed five centrosome zones in the Drosophila centriole. Green, from left to right: immunostaining of Cep135, Spd2, Asl, Cnn, and CP110 antibodies. Red: Dplp antibody. Scale bar, $500 \mathrm{~nm}$.

B Comparison of 3D-SIM, STED, and SMLM in resolving Cep164, a component of distal appendages. Scale bar, $200 \mathrm{~nm}$
A

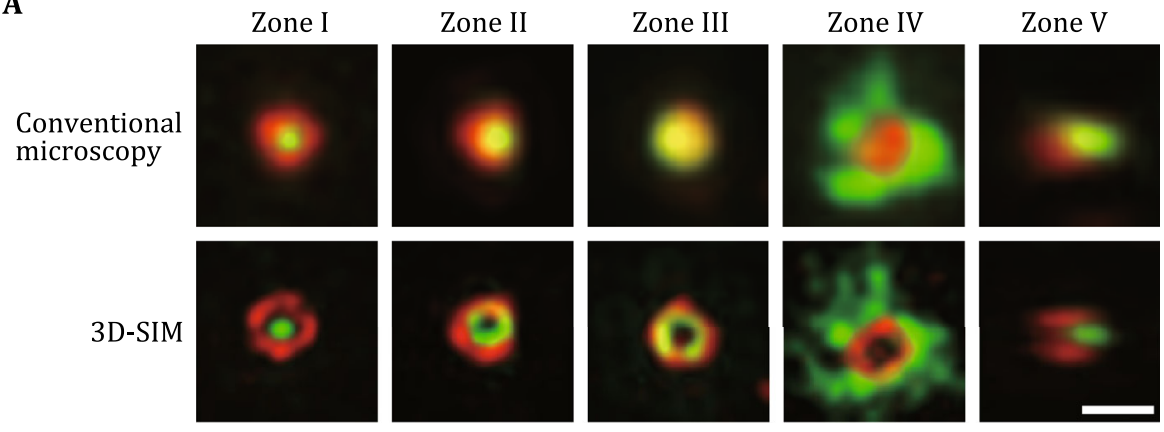

B
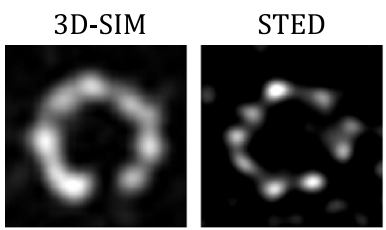

STORM

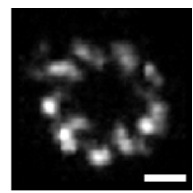

Third, while the above observations are mainly made on the end-on views of centrioles that are located perpendicular to the slides, 3D-SIM takes advantage of the side views of centrioles and dissects the protein distribution along the axis. Combined with the end-on views, the side views can describe a series of proteins involving not only the centriolar and PCM proteins but also the components of distal and subdistal appendages (Huang et al. 2017; Lee et al. 2014; Sydor et al. 2018). For instance, several proteins align along the centrosomal longitudinal axis; from the proximal to distal ends, these proteins are Cep250, Sas6, Cep135, CPAP (homologue of Drosophila Sas4), RTTN (homologue of Drosophila Ana3), POC1B, POC5, and Centrin (Sydor et al. 2018). PPP1R35 (homologue of Drosophila Rcd4) forms a complex with microcephaly protein RTTN, and both of them are required for a proper centriole elongation (Chen et al. 2017; Sydor et al. 2018). At the centriole-cilium interface of multi-ciliated mouse tracheal epithelial cells (MTECs), Cby1 (Drosophila homologue of Chibby) localizes as a ring at the distal end of the mature centrioles, close to the distal end protein Ofd1 and transition zone protein Ahi1 (Lee et al. 2014). Cep164, a component of distal appendages, localizes as a larger ring proximal to Cby1 and Ahi1; Sdccag8, a distal end protein, also localizes proximal to Cby1 (Lee et al. 2014). In another study of U20S cells, the subdistal appendage components Odf2, CCDC68, CCDC120, hNinein, and Cep170 gradually form enlarged rings that are required for hierarchical subdistal appendage assembly and microtubule anchoring in interphase (Huang et al. 2017).

The use of 3D-SIM over the past decade has contributed new knowledge to centrosome research. For instance, in the past, PCM was described to have an amorphous structure that surrounded both centrioles, but today, PCM was revealed to have at least two layers that are recruited to the mother centriole in a temporal manner (Baumann 2012; Fu and Glover 2012; Lawo et al. 2012; Luders 2012; Mennella et al. 2012; Sonnen et al. 2012). 3D-SIM has also provided enormous amounts of data to indicate how centrosome proteins may interact with one another to build the structure.

\section{STIMULATED EMISSION DEPLETION MICROSCOPY (STED)}

STED microscopy is a diffraction-unlimited superresolution microscopy that has attracted wide attention, given its power to achieve super-resolution images without additional data processing, thereby greatly reducing the risk of producing artifacts (Hell and Wichmann 1994; Vicidomini et al. 2018). The resolution enhancement depends on the deactivation of fluorophores in the outer regions of the diffraction-limited excitation focus. In practice, STED uses pairs of synchronized laser pulses for a certain fluorescence dye or protein. The first laser excites the fluorophore to its $\mathrm{ON}$ state, whereas the second laser, called the STED laser, is red-shifted in wavelength and arranged in a donut shape covering the excitation beam. As a result, the fluorescence at the center of the donut remains unaffected, whereas that at the periphery of the excitation spot is switched off via this stimulated emission. STED images can be further processed using deconvolution algorithms that significantly reduce the amount of noise and increase the contrast. The current commercial 3DSTED is based on a confocal system and can achieve a lateral resolution of $50 \mathrm{~nm}$. 3D-STED also allows users 
to select between lateral and axial resolution increase, that is, the axial resolution can ultimately reach $130 \mathrm{~nm}$ at the expense of the $x-y$ resolution.

STED is relatively easy to operate. Commercial systems provide three STED lasers $(592 \mathrm{~nm}, 660 \mathrm{~nm}$, and the pulsed laser at $775 \mathrm{~nm}$ ) that cover the entire spectrum of visible light and are compatible with many conventional dyes or fluorescence proteins. Three-color imaging is routinely possible either by choosing a specific combination of fluorophores that can be deactivated by a single STED laser or a combination that can be deactivated by three STED lasers. In the latter approach, sequential acquisition should be applied starting from the longest wavelength, and imaging can only be performed once because the green-shifted STED laser can lead to photobleaching for the red-shifted fluorophores. Given that STED is based on a confocal system, this technique is more suitable than other super-resolution techniques for imaging thick samples with depths of up to 10 to $15 \mu \mathrm{m}$. However, the acquisition speed of STED is relatively slow for large fields of view. Another drawback of STED is that this approach requires a high laser intensity to make the stimulated emission rate high enough for an effective depletion, and photobleaching may occur during this process. This drawback restricts the application of STED in live cell imaging and 3D sectioning.

STED has been less popular in centrosome research even though its high lateral resolution can resolve Cep164, a distal appendage protein, into nine clusters spaced around a ring with $\sim 300 \mathrm{~nm}$ diameter (Fig. 3B) (Lau et al. 2012; Lee et al. 2014), thereby achieving an actual resolution of below $2 \pi R / 9=105 \mathrm{~nm}$. Compared with 3D-SIM, STED resolves the transition zone proteins Tmem237 and Ahi, the distal end proteins Cby1, Ofd1 and Sdccag8 as puncta around various rings in MTECs, whereas 3D-SIM resolves them as continuous rings (Lee et al. 2014). STED was applied in another study to reveal the architectural map of the transition zone in RPE-1 cells, including both the lateral and longitudinal distribution of eight proteins (Yang et al. 2015). Among these components, transmembrane proteins TMEM67 and TCTN2 are accumulated at the same axial level as MKS1 and RPGRIP1L, and Cep290 is localized at a different axial level closer to the basal body in order to bridge basal body with other transition zone proteins. Cep164 appears at the proximal side of Cep290 along the axis, whereas the distal end protein Centrin is proximal to Cep164. STED has also been used to examine the centrosome linker that connects the two centrosomes from G1/S to the early G2 phase (Vlijm et al. 2018), thereby revealing that Rootletin forms an extended filamentous network with both $\mathrm{N}$ - and
C-termini showing a repeat organization of $75 \mathrm{~nm}$ in RPE-1, primary human umbilical vein endothelial cells (HUVECs), and HCT116 colon cancer cells. Cep68 binds to the C-terminus of Rootletin and exhibits a repeat unit of $75 \mathrm{~nm}$ along the Rootletin filaments to modulate the branching and thickness of the centrosome linker. All these cases demonstrate the power of STED, which has a higher lateral resolution than 3D-SIM, and highlight the need to further explore its application.

\section{SINGLE-MOLECULE LOCALIZATION MICROSCOPY (SMLM)}

Single-molecule localization microscopy (SMLM) is a diffraction-unlimited super-resolution microscopy technique based on single-molecule switching by stochastic excitation (Betzig et al. 2006). It is based on wide-field illumination and can achieve lateral and axial resolutions of 20 and $50 \mathrm{~nm}$, respectively. This technique employs fluorophores that can be induced into a non-emissive dark state, and during each acquisition, only a small fraction of fluorophores is returned to the emissive state and excited. The fluorophores produce sparse fluorescence spots that are diffraction limited. However, the centroid of each excited molecule can be located with high accuracy. By repeating the stochastic excitation and acquisition, more fluorophores in the sample are illuminated and recorded, and a highresolution image is subsequently reconstructed by superposing thousands of single frames. Most SMLMs operate by using the common concept, but differ in the photochemical properties of the fluorophores. For instance, (direct) stochastic optical reconstruction microscopy ((d)STORM) uses organic fluorophores, whereas photoactivated localization microscopy (PALM) uses photoactivatable fluorescent proteins to realize $\mathrm{ON}-\mathrm{OFF}$ switching. The complexity of SMLM lies in its sample preparation, downstream reconstruction, and data analysis, thereby requiring extensive expertise to set up a working system.

SMLM faces several challenges when applied in centrosome research, likely due to the limited amount of fluorophores arranged in a tiny ring-shaped distribution (Keller et al. 2014; Sillibourne et al. 2011; Vlijm et al. 2018). A recent study defined the 3D arrangement of key proteins in the transition zone and found that NPHP1, TMEM231, and RPGRIP1L form nested rings comprised of nine-fold doublets (Shi et al. 2017). Two other studies described the architecture of mammalian centriole distal appendages, a region that protrudes from the microtubule wall with a relatively large diameter (Bowler et al. 2019; Yang et al. 2018). By 
resolving a multiplex of 16 centriole-distal-end components, one study defined a new structural element, the distal appendage matrix, that fills the space between the previously known pinwheel blades (Yang et al. 2018), thereby suggesting that distal appendages may serve as barriers gating the ciliary compartment. The distal appendage matrix contains FBF1 and IFT88, whereas the pinwheel blades mainly comprise Cep83, Cep89, SCLT1, and Cep164. Another study described the initial steps in distal appendage assembly, with CCDC41 initially detectable in G2, SCLT1 accumulated around the prophase, and ANKRD26 appearing in telophase slightly earlier than Cep164 and FBF1 (Bowler et al. 2019). The distal appendages undergo a dramatic ultra-structural reorganization before mitosis and temporarily lose their outer components, while the inner components maintain a nine-fold organization. All these studies have built a roadmap of distal appendages and transition zones with an unprecedented clearness and demonstrate the power of SMLM in revealing the architectural properties of complex cellular structures with nanoscale resolution.

\section{OTHER TECHNIQUES}

\section{D-SIM FRAP}

3D-SIM FRAP performs fluorescence recovery after photobleaching (FRAP) on a fluorescence protein and traces its dynamics by 3D-SIM. This revealed the different characteristics of PCM components Spd2 and Cnn in Drosophila-living embryos (Conduit et al. 2014). After photobleaching, Spd2-GFP fluorescence initially recovers in a toroid shape in zone II and then gradually moves outward, thereby forming dynamic projections that spread away from the centriole. By contrast, GFPCnn fluorescence initially recovers in the central region of the PCM, which is significantly broader than the toroidal region where Spd2-GFP initially recovers, thereby indicating Spd2 and Cnn may not be incorporated into PCM together as part of the same complex. The same group then used two-color 3D-SIM FRAP and found that Sas4 molecules are recruited exclusively to growing daughter centrioles, whereas the PCM components Spd2 and Cnn are recruited only around mother centrioles (Conduit et al. 2015). This finding contrasts that of a previous study which showed that $\mathrm{Cnn}$ is part of the multi-protein S-CAP (Sas4-Cnn-Asl-Dplp) complex that is pre-assembled in the cytosol before being recruited into the centrosome (Gopalakrishnan et al. 2011).

\section{D-SIM and SMLM}

Although the proteins at the outer regions of a centrosome have been extensively studied via superresolution microscopy, proteins at the inner regions remain challenging to resolve. The combination of 3D-SIM and SMLM has been applied on both custom-built microscopes (Rossberger et al. 2013) and commercial instruments, such as the 3D-NSIM/NSTORM Nikon microscope and DeltaVision OMX microscope (Gartenmann et al. 2017; Hamel et al. 2014). The proteins that localize to the inner zones of the centrosome have a limited amount of fluorophores due to their small volume; therefore, the signals collected from a single centrosome cannot be reconstructed reliably into a toroid. To address this problem, one study combined 1200 to 8800 protein localization signals from 40 to 100 centrioles to generate an overall view of the protein distribution (Gartenmann et al. 2017). For each centrosome, a reference channel was initially imaged by 3D-SIM before collecting and aligning SMLM localization data to generate the final image. In this way, the zone I proteins Sas 6 and Ana2 were resolved as toroids with diameters having a standard deviation of 4 to $5 \mathrm{~nm}$. However, this methodology tends to overestimate the average radial distance. The diameter of the $\mathrm{N}$-terminus of Sas6, which is known based on the protein structure and electron cryotomography, is not consistent with the results of the 3D-SIM and SMLM combination (Guichard et al. 2010, 2012; Kitagawa et al. 2011; van Breugel et al. 2011). Therefore, more measurements on proteins with a very small radial distance must be performed to determine the underlying problem of this method.

\section{SMLM and single-particle reconstruction}

Single-particle reconstruction has been widely applied in studying protein structure via cryo-electron microscopy. To study the centrosome protein identity in vitro, a recent study built a computational and analytical framework for reconstructing and co-aligning multiple proteins from 2D-SMLM images (Sieben et al. 2018a, b). This is an alternative way to 3D-SIM and SMLM combination, but in a similar train of thought to combine large amount of centrosome signals for a final image. This method was applied to Cep152 and measured its diameter as $\sim 270 \mathrm{~nm}$, in agreement with the $\sim 242 \mathrm{~nm}$ diameter of SNAP-Cep152 as measured by STED while taking antibody size into account. The Sas6 protein forms an angle to the Cep152 toroid during the pro-centriole formation. However, more proteins should be tested using this method to ensure that the algorithm 
works well for general centrosome proteins before zone I proteins could be readily resolved.

\section{Correlative light electron microscopy}

Super-resolution microscopy has been recently correlated with electron microscopy to determine the area where super-resolved protein signals localize with respect to morphology viewed under electron microscopy. A recent study fitted the signals of centriole distal appendage proteins to the centriole microtubules and appendage electron densities, and revealed the initial assembly of the distal appendages and their reorganization prior to mitosis (Bowler et al. 2019). Electron cryotomography provides $\sim 30$ to $40 \AA$ resolution for several centrosome compartments, including the cartwheel and microtubule triplets/doublets. Combining this approach with super-resolution microscopy offers a powerful method for generating a map of the fine structure of the centrosome with the corresponding protein architecture.

\section{APPLICATIONS BEYOND CENTROSOME}

The past decade not only witnessed the emerging application of super-resolution microscopy in centrosome research but also in the study of many other subcellular organelles and structures, including chromatin, which is mostly resolved by SMLM (Bintu et al. 2018; Birk 2019; Sieben et al. 2018b; Wang et al. 2016; $\mathrm{Xu}$ and Liu 2019), the centromere and kinetochore (Joglekar et al. 2009; Wan et al. 2009; Wynne and Funabiki 2016), the contractile ring (Laplante et al. 2016; McDonald et al. 2017), the nuclear pore complex (Hurt and Beck 2015; Loschberger et al. 2012; Szymborska et al. 2013), and the mitochondria (Brown et al. 2010; Jakobs and Wurm 2014; Jans et al. 2013).

\section{SUMMARY AND PERSPECTIVES}

Almost a century after the discovery under a microscope, the protein architecture of centrosomes remains unknown. The development and application of superresolution microscopy in centrosome research over the past decade has completely changed the way people look at this organelle. After revealing the spatial organization of many proteins, additional questions have emerged, such as what is the full map of zone I components? How does the architecture of centrosomal proteins correlate to the structure we see on an electron microscope or electron cryotomography? How dynamic are centrosomal proteins and how do they change in different cell activities? The continuous development of faster, deeper, and clearer super-resolution microscopy techniques and the integration of super-resolution microscopy with other solutions such as FRAP and correlative electron cryotomography, can introduce additional solutions to the field.

Acknowledgements We thank Dr. Jia Luo for preparing the images shown in Fig. 1, Ms. Yuan Tian for the 3D-SIM and STED images shown in Fig. 3B, and Dr. Tony Yang and Dr. Jung-Chi Liao for the STORM image shown in Fig. 3B. Studies in the Fu Laboratory were supported by the National Natural Science Foundation of China (31722032 and 31771494) and the Ministry of Science and Technology of China (2017YFA0506600), whereas the studies in the Zhang Laboratory were supported by the National Natural Science Foundation of China (31430051 and 31520103906) and the Ministry of Science and Technology of China (2016YFA0500201 and 2016YFA0100501).

\section{Compliance with Ethical Standards}

Conflict of interest Jingyan Fu and Chuanmao Zhang declare that they have no conflict of interest.

Human and animal rights and informed consent This article does not contain any studies with human or animal subjects performed by any of the authors.

Open Access This article is distributed under the terms of the Creative Commons Attribution 4.0 International License (http:// creativecommons.org/licenses/by/4.0/), which permits unrestricted use, distribution, and reproduction in any medium, provided you give appropriate credit to the original author(s) and the source, provide a link to the Creative Commons license, and indicate if changes were made.

\section{References}

Andersen JS, Wilkinson CJ, Mayor T, Mortensen P, Nigg EA, Mann M (2003) Proteomic characterization of the human centrosome by protein correlation profiling. Nature 426:570-574

Balestra FR, Strnad P, Fluckiger I, Gonczy P (2013) Discovering regulators of centriole biogenesis through siRNA-based functional genomics in human cells. Dev Cell 25:555-571

Baumann K (2012) Cell cycle: order in the pericentriolar material. Nat Rev Mol Cell Biol 13:749

Betzig E, Patterson GH, Sougrat R, Lindwasser OW, Olenych S, Bonifacino JS, Davidson MW, Lippincott-Schwartz J, Hess HF (2006) Imaging intracellular fluorescent proteins at nanometer resolution. Science 313:1642-1645

Bintu B, Mateo LJ, Su JH, Sinnott-Armstrong NA, Parker M, Kinrot S, Yamaya K, Boettiger AN, Zhuang X (2018) Super-resolution chromatin tracing reveals domains and cooperative interactions in single cells. Science 362:eaau1783

Birk UJ (2019) Super-resolution microscopy of chromatin. Genes 10:E493

Bowler M, Kong D, Sun S, Nanjundappa R, Evans L, Farmer V, Holland A, Mahjoub MR, Sui H, Loncarek J (2019) Highresolution characterization of centriole distal appendage 
morphology and dynamics by correlative STORM and electron microscopy. Nat Commun 10:993

Brown TA, Fetter RD, Tkachuk AN, Clayton DA (2010) Approaches toward super-resolution fluorescence imaging of mitochondrial proteins using PALM. Methods 51:458-463

Chang CW, Hsu WB, Tsai JJ, Tang CJ, Tang TK (2016) CEP295 interacts with microtubules and is required for centriole elongation. J Cell Sci 129:2501-2513

Chavali PL, Putz M, Gergely F (2014) Small organelle, big responsibility: the role of centrosomes in development and disease. Philos Trans R Soc Lond B Biol Sci 369:20130468

Chen HY, Wu CT, Tang CC, Lin YN, Wang WJ, Tang TK (2017) Human microcephaly protein RTTN interacts with STIL and is required to build full-length centrioles. Nat Commun 8:247

Conduit PT, Richens JH, Wainman A, Holder J, Vicente CC, Pratt MB, Dix CI, Novak ZA, Dobbie IM, Schermelleh L, Raff JW (2014) A molecular mechanism of mitotic centrosome assembly in Drosophila. Elife 3:e03399

Conduit PT, Wainman A, Novak ZA, Weil TT, Raff JW (2015) Reexamining the role of Drosophila Sas-4 in centrosome assembly using two-colour-3D-SIM FRAP. Elife 4:e08483

Demmerle J, Innocent C, North AJ, Ball G, Muller M, Miron E, Matsuda A, Dobbie IM, Markaki Y, Schermelleh L (2017) Strategic and practical guidelines for successful structured illumination microscopy. Nat Protoc 12:988-1010

Dobbelaere J, Josue F, Suijkerbuijk S, Baum B, Tapon N, Raff J (2008) A genome-wide RNAi screen to dissect centriole duplication and centrosome maturation in Drosophila. PLoS Biol 6:e224

Dzhindzhev NS, Tzolovsky G, Lipinszki Z, Schneider S, Lattao R, Fu J, Debski J, Dadlez M, Glover DM (2014) Plk4 phosphorylates Ana2 to trigger Sas6 recruitment and procentriole formation. Curr Biol 24:2526-2532

Dzhindzhev NS, Tzolovsky G, Lipinszki Z, Abdelaziz M, Debski J, Dadlez M, Glover DM (2017) Two-step phosphorylation of Ana2 by Plk4 is required for the sequential loading of Ana2 and Sas6 to initiate procentriole formation. Open Biol 7:170247

Fiolka R, Shao L, Rego EH, Davidson MW, Gustafsson MG (2012) Time-lapse two-color 3D imaging of live cells with doubled resolution using structured illumination. Proc Natl Acad Sci USA 109:5311-5315

Firat-Karalar EN, Stearns T (2014) The centriole duplication cycle. Philos Trans R Soc Lond B Biol Sci 369:20130460

Fu J, Glover DM (2012) Structured illumination of the interface between centriole and pericentriolar material. Open Biol 2:120104

Fu J, Hagan IM, Glover DM (2015) The centrosome and its duplication cycle. Cold Spring Harb Perspect Biol 7:a015800

Fu J, Lipinszki Z, Rangone H, Min M, Mykura C, Chao-Chu J, Schneider S, Dzhindzhev NS, Gottardo M, Riparbelli MG, Callaini G, Glover DM (2016) Conserved molecular interactions in centriole-tocentrosome conversion. Nat Cell Biol 18:87-99

Gartenmann L, Wainman A, Qurashi M, Kaufmann R, Schubert S, Raff JW, Dobbie IM (2017) A combined 3D-SIM/SMLM approach allows centriole proteins to be localized with a precision of approximately 4-5 nm. Curr Biol 27:1054-1055

Godinho SA, Pellman D (2014) Causes and consequences of centrosome abnormalities in cancer. Philos Trans R Soc Lond B Biol Sci 369:20130467

Gopalakrishnan J, Mennella V, Blachon S, Zhai B, Smith AH, Megraw TL, Nicastro D, Gygi SP, Agard DA, Avidor-Reiss T (2011) Sas-4 provides a scaffold for cytoplasmic complexes and tethers them in a centrosome. Nat Commun 2:359
Goshima G, Wollman R, Goodwin SS, Zhang N, Scholey JM, Vale RD, Stuurman N (2007) Genes required for mitotic spindle assembly in Drosophila S2 cells. Science 316:417-421

Guichard P, Chretien D, Marco S, Tassin AM (2010) Procentriole assembly revealed by cryoelectron tomography. EMBO J 29:1565-1572

Guichard P, Desfosses A, Maheshwari A, Hachet V, Dietrich C, Brune A, Ishikawa T, Sachse C, Gonczy P (2012) Cartwheel architecture of Trichonympha basal body. Science 337:553

Gustafsson MG (2000) Surpassing the lateral resolution limit by a factor of two using structured illumination microscopy. J Microsc 198:82-87

Gustafsson MG, Shao L, Carlton PM, Wang CJ, Golubovskaya IN, Cande WZ, Agard DA, Sedat JW (2008) Three-dimensional resolution doubling in wide-field fluorescence microscopy by structured illumination. Biophys J 94:4957-4970

Hamel V, Guichard P, Fournier M, Guiet R, Fluckiger I, Seitz A, Gonczy P (2014) Correlative multicolor 3D SIM and STORM microscopy. Biomed Opt Express 5:3326-3336

Heintzmann R, Huser T (2017) Super-resolution structured illumination microscopy. Chem Rev 117:13890-13908

Hell SW, Wichmann J (1994) Breaking the diffraction resolution limit by stimulated emission: stimulated-emission-depletion fluorescence microscopy. Opt Lett 19:780-782

Huang N, Xia Y, Zhang D, Wang S, Bao Y, He R, Teng J, Chen J (2017) Hierarchical assembly of centriole subdistal appendages via centrosome binding proteins CCDC120 and CCDC68. Nat Commun 8:15057

Hurt E, Beck M (2015) Towards understanding nuclear pore complex architecture and dynamics in the age of integrative structural analysis. Curr Opin Cell Biol 34:31-38

Izquierdo D, Wang WJ, Uryu K, Tsou MF (2014) Stabilization of cartwheel-less centrioles for duplication requires CEP295mediated centriole-to-centrosome conversion. Cell Rep 8:957-965

Jakobs S, Wurm CA (2014) Super-resolution microscopy of mitochondria. Curr Opin Chem Biol 20:9-15

Jakobsen L, Vanselow K, Skogs M, Toyoda Y, Lundberg E, Poser I, Falkenby LG, Bennetzen M, Westendorf J, Nigg EA, Uhlen M, Hyman AA, Andersen JS (2011) Novel asymmetrically localizing components of human centrosomes identified by complementary proteomics methods. EMBO J 30:1520-1535

Jans DC, Wurm CA, Riedel D, Wenzel D, Stagge F, Deckers M, Rehling P, Jakobs S (2013) STED super-resolution microscopy reveals an array of MINOS clusters along human mitochondria. Proc Natl Acad Sci USA 110:8936-8941

Joglekar AP, Bloom K, Salmon ED (2009) In vivo protein architecture of the eukaryotic kinetochore with nanometer scale accuracy. Curr Biol 19:694-699

Keller D, Orpinell M, Olivier N, Wachsmuth M, Mahen R, Wyss R, Hachet V, Ellenberg J, Manley S, Gonczy P (2014) Mechanisms of HsSAS-6 assembly promoting centriole formation in human cells. J Cell Biol 204:697-712

Kitagawa D, Vakonakis I, Olieric N, Hilbert M, Keller D, Olieric V, Bortfeld M, Erat MC, Fluckiger I, Gonczy P, Steinmetz MO (2011) Structural basis of the 9-fold symmetry of centrioles. Cell 144:364-375

Laplante C, Huang F, Tebbs IR, Bewersdorf J, Pollard TD (2016) Molecular organization of cytokinesis nodes and contractile rings by super-resolution fluorescence microscopy of live fission yeast. Proc Natl Acad Sci USA 113:5876-5885

Lau L, Lee YL, Sahl SJ, Stearns T, Moerner WE (2012) STED microscopy with optimized labeling density reveals 9-fold arrangement of a centriole protein. Biophys J 102:2926-2935

Lawo S, Hasegan M, Gupta GD, Pelletier L (2012) Subdiffraction imaging of centrosomes reveals higher-order organizational 
features of pericentriolar material. Nat Cell Biol 14:1148-1158

Lee YL, Sante J, Comerci CJ, Cyge B, Menezes LF, Li FQ, Germino GG, Moerner WE, Takemaru K, Stearns T (2014) Cby1 promotes Ahi1 recruitment to a ring-shaped domain at the centriolecilium interface and facilitates proper cilium formation and function. Mol Biol Cell 25:2919-2933

Loschberger A, van de Linde S, Dabauvalle MC, Rieger B, Heilemann M, Krohne G, Sauer M (2012) Super-resolution imaging visualizes the eightfold symmetry of gp210 proteins around the nuclear pore complex and resolves the central channel with nanometer resolution. J Cell Sci 125:570-575

Luders J (2012) The amorphous pericentriolar cloud takes shape. Nat Cell Biol 14:1126-1128

McDonald NA, Lind AL, Smith SE, Li R, Gould KL (2017) Nanoscale architecture of the Schizosaccharomyces pombe contractile ring. Elife 6:e28865

Mennella V, Keszthelyi B, McDonald KL, Chhun B, Kan F, Rogers GC, Huang B, Agard DA (2012) Subdiffraction-resolution fluorescence microscopy reveals a domain of the centrosome critical for pericentriolar material organization. Nat Cell Biol 14:1159-1168

Muller H, Schmidt D, Steinbrink S, Mirgorodskaya E, Lehmann V, Habermann K, Dreher F, Gustavsson N, Kessler T, Lehrach H, Herwig R, Gobom J, Ploubidou A, Boutros M, Lange BM (2010) Proteomic and functional analysis of the mitotic Drosophila centrosome. EMBO J 29:3344-3357

Nigg EA, Holland AJ (2018) Once and only once: mechanisms of centriole duplication and their deregulation in disease. Nat Rev Mol Cell Biol 19:297-312

Rossberger S, Best G, Baddeley D, Heintzmann R, Birk U, Dithmar S, Cremer C (2013) Combination of structured illumination and single molecule localization microscopy in one setup. J Opt 15:094003

Saurya S, Roque H, Novak ZA, Wainman A, Aydogan MG, Volanakis A, Sieber B, Pinto DM, Raff JW (2016) Drosophila Ana1 is required for centrosome assembly and centriole elongation. J Cell Sci 129:2514-2525

Shao L, Kner P, Rego EH, Gustafsson MG (2011) Super-resolution 3D microscopy of live whole cells using structured illumination. Nat Methods 8:1044-1046

Shi X, Garcia G 3rd, Van De Weghe JC, McGorty R, Pazour GJ, Doherty D, Huang B, Reiter JF (2017) Super-resolution microscopy reveals that disruption of ciliary transition-zone architecture causes Joubert syndrome. Nat Cell Biol 19:1178-1188

Sieben C, Banterle N, Douglass KM, Gonczy P, Manley S (2018a) Multicolor single-particle reconstruction of protein complexes. Nat Methods 15:777-780

Sieben C, Douglass KM, Guichard P, Manley S (2018b) Superresolution microscopy to decipher multi-molecular assemblies. Curr Opin Struct Biol 49:169-176

Sillibourne JE, Specht CG, Izeddin I, Hurbain I, Tran P, Triller A, Darzacq X, Dahan M, Bornens M (2011) Assessing the localization of centrosomal proteins by PALM/STORM nanoscopy. Cytoskeleton (Hoboken) 68:619-627

Sir JH, Barr AR, Nicholas AK, Carvalho OP, Khurshid M, Sossick A, Reichelt S, D'Santos C, Woods CG, Gergely F (2011) A primary microcephaly protein complex forms a ring around parental centrioles. Nat Genet 43:1147-1153

Sonnen KF, Schermelleh L, Leonhardt H, Nigg EA (2012) 3Dstructured illumination microscopy provides novel insight into architecture of human centrosomes. Biol Open 1:965-976

Sydor AM, Coyaud E, Rovelli C, Laurent E, Liu H, Raught B, Mennella V (2018) PPP1R35 is a novel centrosomal protein that regulates centriole length in concert with the microcephaly protein RTTN. Elife 7:e37846

Szymborska A, de Marco A, Daigle N, Cordes VC, Briggs JA, Ellenberg J (2013) Nuclear pore scaffold structure analyzed by super-resolution microscopy and particle averaging. Science 341:655-658

Tsuchiya Y, Yoshiba S, Gupta A, Watanabe K, Kitagawa D (2016) Cep295 is a conserved scaffold protein required for generation of a bona fide mother centriole. Nat Commun 7:12567

Uzbekov R, Alieva I (2018) Who are you, subdistal appendages of centriole? Open Biol 8:180062

van Breugel M, Hirono M, Andreeva A, Yanagisawa HA, Yamaguchi S, Nakazawa Y, Morgner N, Petrovich M, Ebong IO, Robinson CV, Johnson CM, Veprintsev D, Zuber B (2011) Structures of SAS-6 suggest its organization in centrioles. Science 331:1196-1199

Vicidomini G, Bianchini P, Diaspro A (2018) STED super-resolved microscopy. Nat Methods 15:173-182

Vlijm R, Li X, Panic M, Ruthnick D, Hata S, Herrmannsdorfer F, Kuner T, Heilemann M, Engelhardt J, Hell SW, Schiebel E (2018) STED nanoscopy of the centrosome linker reveals a CEP68organized, periodic rootletin network anchored to a C-Nap1 ring at centrioles. Proc Natl Acad Sci USA 115:2246-2253

Wan X, O'Quinn RP, Pierce HL, Joglekar AP, Gall WE, DeLuca JG, Carroll CW, Liu ST, Yen TJ, McEwen BF, Stukenberg PT, Desai A, Salmon ED (2009) Protein architecture of the human kinetochore microtubule attachment site. Cell 137:672-684

Wang S, Su JH, Beliveau BJ, Bintu B, Moffitt JR, Wu CT, Zhuang X (2016) Spatial organization of chromatin domains and compartments in single chromosomes. Science 353:598-602

Wu Y, Shroff H (2018) Faster, sharper, and deeper: structured illumination microscopy for biological imaging. Nat Methods 15:1011-1019

Wynne DJ, Funabiki H (2016) Heterogeneous architecture of vertebrate kinetochores revealed by three-dimensional superresolution fluorescence microscopy. Mol Biol Cell 27:3395-3404

Xu J, Liu Y (2019) A guide to visualizing the spatial epigenome with super-resolution microscopy. FEBS J. https://doi.org/10. 1111/febs.14938

Xu X, Huang S, Zhang B, Huang F, Chi W, Fu J, Wang G, Li S, Jiang Q Zhang C (2017) DNA replication licensing factor Cdc6 and Plk4 kinase antagonistically regulate centrosome duplication via Sas-6. Nat Commun 8:15164

Yang TT, Su J, Wang WJ, Craige B, Witman GB, Tsou MF, Liao JC (2015) Superresolution pattern recognition reveals the architectural map of the ciliary transition zone. Sci Rep 5:14096

Yang TT, Chong WM, Wang WJ, Mazo G, Tanos B, Chen Z, Tran TMN, Chen YD, Weng RR, Huang CE, Jane WN, Tsou MB, Liao JC (2018) Super-resolution architecture of mammalian centriole distal appendages reveals distinct blade and matrix functional components. Nat Commun 9:2023

Zhang B, Zhang T, Wang G, Wang G, Chi W, Jiang Q Zhang C (2015) GSK3beta-Dzip1-Rab8 cascade regulates ciliogenesis after mitosis. PLoS Biol 13:e1002129

Zhang B, Wang G, Xu X, Yang S, Zhuang T, Wang G, Ren H, Cheng SY, Jiang Q Zhang C (2017) DAZ-interacting protein 1 (Dzip1) phosphorylation by polo-like kinase 1 (Plk1) regulates the centriolar satellite localization of the BBSome protein during the cell cycle. J Biol Chem 292:1351-1360

Zhang B, Zhuang T, Lin Q Yang B, Xu X, Xin G, Zhu S, Wang G, Yu B, Zhang T, Jiang Q Zhang C (2019) Patched1-ArhGAP36-PKAInversin axis determines the ciliary translocation of smoothened for sonic hedgehog pathway activation. Proc Natl Acad Sci USA 116:874-879 